\title{
A case study report of denovo lymph node occurance of myeloid sarcoma
}

\author{
Dr. Raveendra Naik ${ }^{1}$, V B Sankara Rao², T V Ramesh ${ }^{3}$, Y.S Sarma ${ }^{4}$, Keerthi Priya Konkay ${ }^{5}$ \\ ${ }^{1}$ Dr. D. Raveendra Naik MBBS Jr.Resident, Department of Paediatrics, ${ }^{2}$ Dr. J V B Sankara Rao, MBBS Jr. Resident , \\ Department of Paediatrics, ${ }^{3}$ Dr. T V Ramesh, MD (Paediatrics), Professor and Head of paediatrics, ${ }^{4}$ Dr. Y.S Sarma, MD, \\ DM (oncology), Professor of Medicine, ${ }^{5}$ Dr. Keerthi Priya Konkay, MBBS Jr. Resident, Department of Paediatrics. All \\ authors are affiliated with GSL Medical College Rajahmundry, India.
}

Address for correspondence: Dr. D. Raveendra Naik, Email: drdrnaik@gmail.com

\begin{abstract}
Myeloid sarcoma (MS) as a rare localized solid tumor mass consists of myeloblasts or immature myeloid cells was found in an extramedullary site of a 4 year male child who was suffering with fever, abdominal distension since 4 months and swelling of legs from 1month . On examining pallor, bilateral cervical and inguinal lymphadenopathy, bilateral pitting pedal edema, Hepatosplenomegaly \& ascites found. On investigation anemia with leukocytosis, thrombocytopenia was found. USG abdomen showed multiple non necrotic lymphnodes. Bone marrow aspiration suggestive of JMML, lymphnode biopsy showed myeloid sarcoma and was confirmed by immune histochemistry (IHC) markers LCA, KP1(CD68), myeloperoxidase are positive.Immunophenotyping is useful in differentiating myeloid sarcoma from lymphoma and leukaemia. Child was treated with 3/7 regimen of cytoarabinoside, daunorubicin.
\end{abstract}

Key words: Lymphnode Biopsy, Immunohistochemistry, KP1 (CD68), Myeloid Sarcoma.

\section{Introduction}

In world health organization (WHO) 2007 classification of lymphoid and hematopoietic neoplasms has described myeloid sarcoma (MS) as a rare localized solid tumor mass consisting of myeloblasts or immature myeloid cells in an extramedullary site [1]. MS typically occurs in the later decades of life with median age of 56 years; ranging from, 1 month to 89 years [2]. MS may occur as denovo or concurrently with acute myeloid leukemia (AML), myeloproliferative disorder (MPD) or myelodysplastic syndrome (MDS) or relapse of AML [3] or after allogenic stem cell transplantation [4]. MS occur any part of body most commonly involved site are subperiosteal bone structures of the skull, paranasal sinuses, the sternum, ribs, vertebrae and pelvis, lymph nodes and skin .other rare sites reported in the literature include the pancreas, heart, brain, mouth, breast, gastrointestinal and biliary tract, prostate, urinary bladder and gynecologic tract [5]. MS symptoms and signs depend on site of location. Chemotherapy or hematopoietic cell transplantation is often considered the front-line treatment for MS.

Manuscript received: $11^{\text {th }}$ Dec 2015

Reviewed: 21th Dec 2015

Author Corrected; 02th Jan 2016

Accepted for Publication: $12^{\text {th }}$ Jan 2016

\section{Case Report}

A 4 years male child was presented with complaint of fever, abdominal distension since 4 months and swelling of both legs of from 1month. Fever is intermittent, high grade, not associated with chills and rigors. No cold and cough, burning micturition, vomiting, loose watery stools. No past history of congenital heart disease, contact with tuberculosis, recurrent respiratory tract infection, jaundice, connective tissue disorders. Only child was born out of non consanguineous marriage. No similar complaints in family.

On examining pallor generalized lymphadenopthy was found .Bilateral cervical lymphnodes of both anterior \& posterior groups of size $5 \times 3 \mathrm{~cm}$, bilateral Inguinal lymph nodes of size $4 X 3 \mathrm{~cm}$ firm, mobile, not matted. No cyanosis, icterus, clubbing. Bilateral pedal edema was found Hepatomegaly $(3 \mathrm{~cm}$ below right costal margin), grade III splenomegaly preset. Free fluid is found. Respiratory, CVS, CNS are found to be normal. On investigation hemoglobin was $5.9 \mathrm{gms}$, total leukocytes 44,000/cumm, platelet count 30000, peripheral smear shows leukocytosis with atypical cells/ 
blasts $3 \%$, myelocytes $3 \%$, metamyelocytes $4 \%$, neutrophils $72 \%$, lymphocytes $18 \%$. Ultrasound abdomen shows multiple oval shaped isoechoic non necrotic lymphnodes are noted in periportal, peripancreatic, mesenteric, parailiac regions largest measuring 9-11 mm size. Hepatosplenomegaly and, ascitis are found. Retroviral test is non reactive, Mantoux is negative. Bone Marrow Aspiration (BMA) shows $\mathrm{M}$ :E ratio 1.7:1, increased megaloblastoid erythropoeisis, myelopoesis with predominant cells includes blast cells $12 \%$, promyelocytes $8 \%$, myelocytes $17 \%$, metamyeloctes $5 \%$, band forms \& neutrophils $16 \%$ lymphocytes 5 , erythroid cells $37 \%$, megakaryocytes reduced with dysmegakaryosis with multiple form of nucleoli suggestive of juvenile myelomonocytic leukemia (JMML).

Inguinal lymphnode biopsy showed there are few presereved follicles with expansion of inter follicular \& parafollicular area, infilterated by medium to large sized cells with irregular nuclei, prominent nucleoli. ImmunoHistochemistry was positive for LCA, KP1(CD68), myeloperoxidase. Final diagnosis of extramedullary lymphnode manifestion of monocytic type of myeloid sarcoma was made.

\section{Discussion}

Myeloid sarcoma is also known as chloroma, granulocytic sarcoma. Clinical presentation depends on the site of involvement. Myeloid sarcomas is found to be one of the condition with incidence as denovo lymphnode manifestation in whom a typical form of AML may occur after an interval of weeks, months or even years. Rarely no leukemia develops.(1.1\%), In association with AML, CMPD or MDS (3-9\%) manifestation of relapse is found to be in patients previously treated for primary or secondary acute leukemia [6] .

An extramedullary relapse after allogenic stem cell transplantation with interval of 4-56 month $(<1 \%)$ [7]. No age group is immune; however, Myeloid sarcoma has a predilection for males (male-to-female ratio, 1.1:2. The pathogenesis mechanism of isolated myeloid sarcoma remains unknown. Histopathologically MS classified based on degree of maturation divided into 1. A blastic variant with predominance of myeloblasts.

2. An immature variant with a mix of myeloblasts and promyelocytes.

3. A differentiated variant with promyelocytes and more mature granulocyte.
MS based on most abundant cell into granulocytic, monoblastic, or myelomonocytic [8]. Tissue biopsy specimen IHC is highly effective establishing diagnosis of myeloid sarcoma. Reactivity with various antibodies depends in part on the degree of differentiation of neoplastic cells most cases of myeloid sarcoma are positive for lysozyme, CD117(c-kit) $>90 \% \mathrm{KP}-1$ (100\%) PG-M1(51\%), myeloperoxidase (80-90\%) LCA (CD45) $70-80 \%$ of myeloid sarcoma [9].

Others antibodies react with MS are CD99 and TdT, CD34 neutrophil elastase, CD15 and Mac 387, CD56, CD20. However B-cell transcription factor PAX5/BSAP is commonly expressed in myeloid neoplasm associated with $\mathrm{t}(8,21) \quad(\mathrm{q} 22,22)$ Cytogenetically involved translocations in MS are $\mathrm{t}(8,22)$ (q22 22), inv16, (p13q21) and monocytic lineage as well as $\mathrm{t}(9,22)$ rarely monosomy 7 trisomy 8 have been detected in myeloid sarcoma.

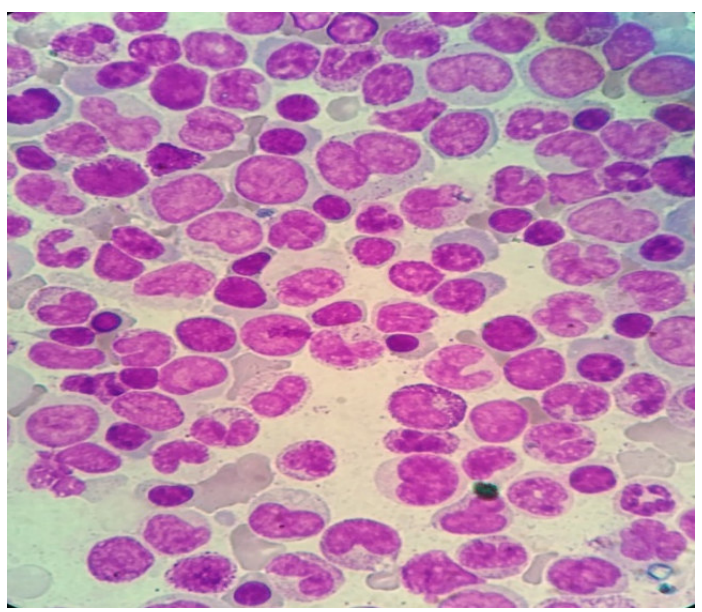

Fig 1: Bone marrow aspiration shows atypical lymphocytes

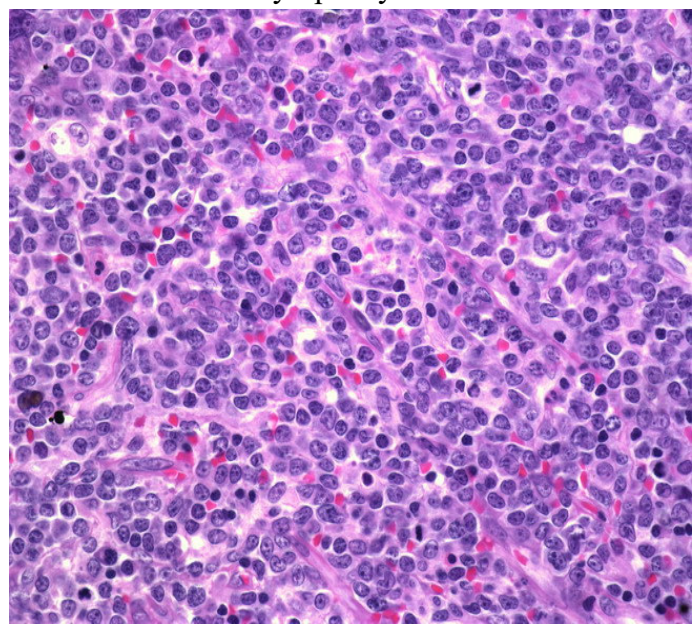

Fig 2: Lymph node biopsy matured lymphocytes With hpersegmented nuetrophils, myelocytes metamyelocytes And blasts 


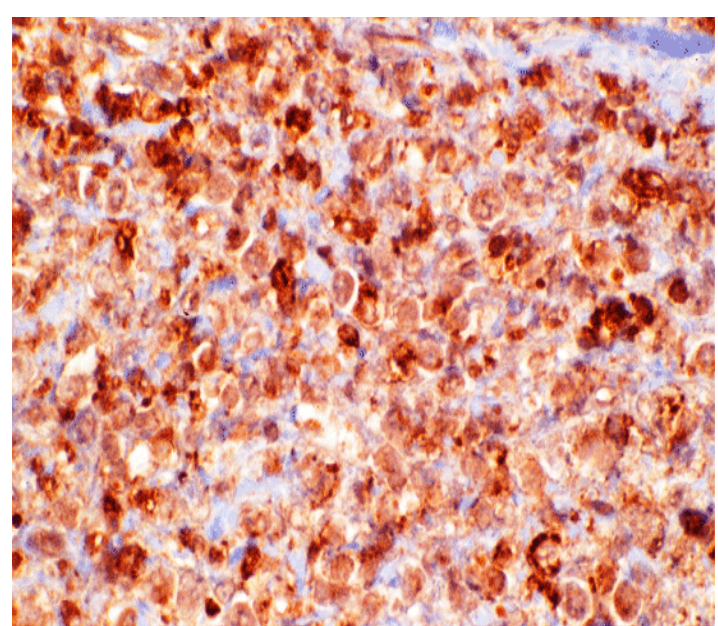

Fig 3: Lymph node biopsy shows CD-68(KP-1) positive

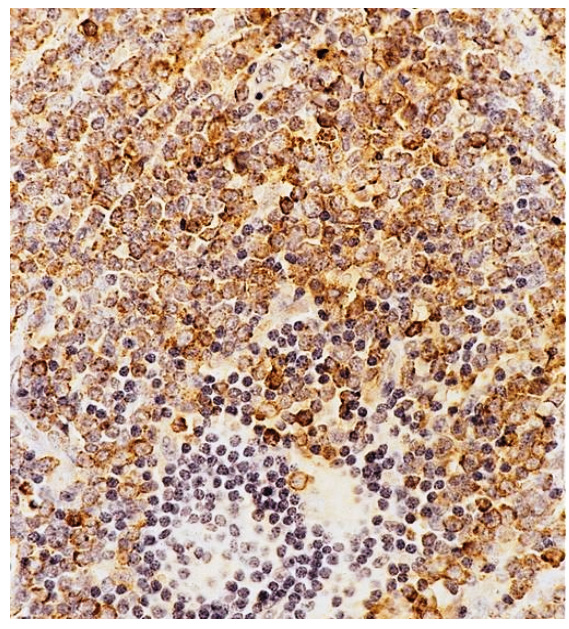

Fig 4: Lymph node biopsy shows MPO (myeloperoxidase) positive

\section{Conclusion}

Denovo lymph node Myeloid sarcoma - is a rare presentation, Diagnosis of it is often missed initial stages of The disease. But immunophenotyping is very useful in establishing diagnosis of this disease.

It needs to be further evaluated by molecular studies for prognosis. And for intensive study AML like therapy is needed.
Abbreviation
MS : Myeloid Sarcoma
AML : Acute Myeloid Luekemia
JMML: Juvenile Myelo Monocytic Luekemia
CMPD: Chronic Myelo Proliferative Disorder
MDS : Myelo Displastic Syndrome

\section{References}

1. Brunning RD, Matutes E, Flandrin G, Vardiman J, Bennett J, Head D, et al. Acute myeloid leukaemia not otherwise categorised. In: Jaffe ES, Stein H, Vardiman JW, editors. Tumors of Hematopoietic and Lymphoid Tissues. Lyon: IARC Press; 2001. pp. 104-5.

2. Pileri S.A., Ascani S., Cox M., Campidelli C., Bacci F., Piccioli M., et al. (2007) Myeloid sarcoma: Clinicopathologic, phenotypic and cytogenetic analysis of 92 adult patients. Leukemia 21: 340-350.

3. Tsimberidou AM, Kantarjian HM, Wen S, Keating MJ, O'Brien S, Brandt M, Pierce S, Freireich EJ, Medeiros LJ, Estey E. Myeloid sarcoma is associated with superior event-free survival and overall survival compared with acute myeloid leukemia. Cancer. 2008 Sep 15;113(6):1370-8. doi: 10.1002/cncr.23691.

4. Cunningham I. Extramedullary sites of leukemia relapse after transplant. Leuk Lymphoma. 2006 Sep; 47(9):1754-67.

5. Audouin J, Comperat E, Le Tourneau A, CamilleriBroët S, Adida C, Molina T, Diebold J. Myeloid sarcoma: clinical and morphologic criteria useful for diagnosis. Int J Surg Pathol. 2003 Oct; 11(4):271-82.

6. Rappaport H. Tumors of the hematopoietic system. Armed Forces Inst Pathol 1966; 3:241-43.

7. Szomor A, Passweg JR, Tichelli A, Hoffmann T, Speck B, Gratwohl A. Myeloid leukemia and myelodysplastic syndrome relapsing as granulocytic sarcoma (chloroma) after allogeneicbone marrow transplantation. Ann Hematol. 1997 Nov-Dec; 75(56):239-41.

8.Lan TY, Lin DT, Tien HF, Yang RS, Chen CY, Wu K. Prognostic factors of treatment outcomes in patients with granulocytic sarcoma. Acta Haematol. 2009;122(4):238-46. doi: 10.1159/000253592. Epub 2009 Oct 30 . 
9. Maeng H, Cheong JW, Lee ST, et al.Isolated extramedullary relapse of acute myelogenous leukemia as a uterine granulocytic sarcoma in an allogeneic hematopoietic stem cell transplantation recipient. Yonsei Med J.2004; 45:330-3.

\section{How to cite this article?}

Dr. Raveendra Naik, J V B Sankara Rao, T V Ramesh, Y.S Sarma, Keerthi Priya Konkay. A case study report of denovo lymph node occurance of myeloid sarcoma.Pediatr Rev: Int J Pediatr Res 2016;3(1):73-76.doi:10.17511/ijpr.2016.i01.14 